\title{
Expert Opinion on Restoration of Pediatric Pulmonology Services During the SARS-CoV-2 Pandemic
}

\author{
Kiran Kumar Banothu, ${ }^{1}$ Javeed Iqbal Bhat, ${ }^{2}$ Rashmi Ranjan Das, ${ }^{3}$ Nitin Dhochak, ${ }^{1}$ Jagat Jeevan \\ Ghimire, ${ }^{1}$ Jagdish P Goyal, ${ }^{4}$ Krishna Mohan Gulla, ${ }^{3}$ Samriti Gupta, ${ }^{5}$ Kana Ram Jat, ${ }^{1}$ Sushil K Kabra, ${ }^{1}$ \\ Pawan Kalyan, ${ }^{6}$ Arvind Kumar, ${ }^{7}$ Prawin Kumar, ${ }^{4}$ ViJay Kumar, ${ }^{1}$ Rakesh Lodha, ${ }^{1}$ Joseph L Mathew, ${ }^{8}$ \\ Amit Pathania, ${ }^{\mathbf{1}}$ Vinod H Ratageri, ${ }^{\mathbf{9}}$ Varinder Singh ${ }^{\mathbf{1 0}}$ ANd Sagar WarankaR ${ }^{\mathbf{1 1}}$ (Authors are listed in \\ alphabetical order) \\ From Department of Pediatrics, ${ }^{1}$ All India Institute of Medical Sciences (AIIMS), New Delhi; ${ }^{2}$ Sher-i-Kashmir Institute of Medical \\ Sciences, Srinagar, Jammu and Kashmir; ${ }^{3}$ AIIMS, Bhubaneswar, Odisha; ${ }^{4}$ AIIMS, Jodhpur, Rajasthan; ${ }^{5}$ Dr Rajendra Prasad \\ Government Medical College, Tanda, Himachal Pradesh; ${ }^{6}$ Dr Pinnamaneni Siddhartha Institute of Medical Sciences \& Research \\ Foundation, Vijayawada, Andhra Pradesh; ${ }^{7}$ Army Institute of Cardio-Thoracic Sciences, Pune, Maharashtra; ${ }^{8}$ Postgraduate \\ Institute of Medical Education and Research (PGIMER), Chandigarh; ${ }^{9}$ Karnataka Institute of Medical Sciences, Hubli, Karnataka; \\ ${ }^{10}$ Kalawati Saran Children's Hospital, Lady Hardinge Medical College, New Delhi; and ${ }^{11}$ Consultant, Mumbai, Maharashtra; \\ India. \\ Correspondence to: Dr Kana Ram Jat, Department of Pediatrics, All India Institute of Medical Sciences, New Delhi 110 029, India. \\ drkanaram@gmail.com
}

Published online: September 16, 2020; PII: S097475591600249

$\mathrm{T}$ he severe acute respiratory syndrome coronavirus-2 (SARS-CoV-2) pandemic has had an unprecedented impact on public health and healthcare services delivery worldwide. There are many challenges in resuming non-coronavirus disease (COVID-19) services like pediatric pulmonology services. These include, among others, difficulties in restoration of resources diverted for COVID-19 care, risk of overwhelming of the services due to backlog, and apprehension among the health care workers of acquiring the disease.

In the absence of evidence-based guidelines on restarting pediatric pulmonology services in a pandemic situation, this consensus statement has been designed to provide guidance to healthcare professionals and/or institutions for restoration of pediatric pulmonology services during the SARS-CoV-2 pandemic and postpandemic phase.

\section{PROCESS}

A group of specialists with expertise and experience in pediatric pulmonology, across India was identified and a catalogue of services/procedures to be restarted was listed. The list included clinical care of children with respiratory problems in various settings, therapeutic procedures, and diagnostic procedures (Table I). The group was subdivided to prepare a position statement on various services and procedures based on available literature. This was collated together by the coordinator for review by another set of experts. The internal peer review was shared with the contributors for preparing the revised version which was again subjected to the internal peer review followed by a circulation to the whole group to arrive at the final recommendations.

The target audience for this consensus statement includes healthcare professionals who have been dealing with pediatric respiratory services prior to the COVID-19 pandemic. This consensus statement is not intended to be used for the care of children with confirmed COVID-19, even if asymptomatic.

\section{RECOMMENDATIONS}

The position statement suggests following general measures to prevent the transmission of SARS-CoV-2 $[1,2]$.

Reduce hospital visits: The hospital visits should be prioritized for those with unresolved diagnostic or therapeutic issues of urgent or semi-urgent nature. The triaging of needs can be done by using tele-health consultations for all cases (follow-up as well as new) [3]. Routine visits for follow-up in stable patients should be deferred and staggered or preferably replaced by a telehealth consultation. The visits for collection of medicines should be staggered too by making administrative arrangement so that the caregiver can directly collect the 
Table I List of Services Along with Risk of Infection, Timing of Resumption, and Required Personal Protection

\begin{tabular}{|c|c|c|c|}
\hline Service/ procedure & Risk* & Timing of resumption ${ }^{\#}$ & PPE level` \\
\hline \multicolumn{4}{|l|}{ Clinical care } \\
\hline Evaluation in out-patient setting & 1 & $\mathrm{I}$ & Standard \\
\hline In-patient care & 2 & I & Standard \\
\hline Emergency care & 3 & I & Full \\
\hline Care of children with stable chronic respiratory diseases & 1 & I & Standard \\
\hline \multicolumn{4}{|l|}{ Therapeutic procedures } \\
\hline Use of nebulizer (any type) & 3 & I & Full \\
\hline Use of metered dose inhaler with spacer & 1 & I & Standard \\
\hline Use of heated humidified high flow nasal cannula & 3 & I & Full \\
\hline Use of ventilator & 3 & I & Full \\
\hline Airway clearance techniques & 3 & I & Full \\
\hline \multicolumn{4}{|l|}{ Diagnostic Procedures } \\
\hline Gastric aspirate and induced sputum & 3 & II & Full \\
\hline Throat/nasal swab, nasopharyngeal aspirate & 3 & $\mathrm{III}^{t}$ & Full \\
\hline Pulmonary function test & 2 & II & Standard \\
\hline Flexible bronchoscopy & 3 & II & Full \\
\hline Nasal NO/FeNO & 2 & II & Standard \\
\hline High speed video microscopy and electron microscopy & 3 & II & Full \\
\hline Sweat testing and aquagenic wrinkling & 1 & I & Standard \\
\hline Imaging procedures & 1 & I & Standard \\
\hline Exercise testing & 2 & III & Standard \\
\hline Infant PFT & 2 & III & Standard \\
\hline Tuberculin skin test and skin prick test & 1 & TST: I, SPT: III & Standard \\
\hline
\end{tabular}

drugs from the pharmacy without needing to visit the doctor. For those identified to need a direct contact during a tele-consultation, should be given appointment, and preparatory advice about safety and precautions to be followed during the visit. The outcome of the visits can be maximised by advising the basic investigations to be done locally before visit and planning the specialized tests or procedures anticipated on the day of visit as far as feasible.

Reduce chances of cross-infection during visit: Reduce crowding and time spent in health care facility by previsit tele consultation, staggered appointments, and by restricting the number of accompanying attendant(s) with children. Screening all cases for active influenza like illness (ILI) on the day of visit at hospital entry, and segregating those with active symptoms to separate designated areas for COVID suspects is important. Advise patients and attendants to wear mask correctly, maintain social distancing, follow the cough/sneezing etiquette, and hand sanitization. Frequent sanitization/ disinfection of patient care area; and use of appropriate personal protective equipment (PPE) by health care workers should be ensured.

In the outpatient area, restrict the consult to one patient entering at a time and perform hand hygiene and sanitation of equipment (stethoscope, pen, etc.) before, after and between consultations.

Precautions while admitting children with respiratory problems: Prioritize in-patient care of children for those with a definitive need e.g. prophylactic IVIG may be prioritized as there is no alternative therapy while the admissions for pulse steroids could be restricted by using oral steroids instead till the pandemic shows flattening of curve. Similarly, less severe exacerbation of suppurative lung diseases may be advised oral antibiotics at home or injectables at nearby health facility. 
In the inpatient area, adequate spacing should be maintained between beds. In the absence of negative pressure rooms, exhaust fans could be used in rooms along with air-conditioning and if required, windows/ doors can be left open for better air exchange. Single use/ disposable/ dedicated equipment should be used and when this is not feasible, thorough cleaning and disinfection of equipment should be ensured before using on any other child. Reduce time of physical rounds by discussing details beforehand. Try to reduce hospital stay of patients to the minimum.

Pre-testing for COVID: In case the facilities and capacities exist, particularly in non-urgent situations, testing for SARS-CoV-2 using RT-PCR or CBNAAT can be done before hospitalization and before all aerosol generating procedures like gastric aspirate, induced sputum, and flexible bronchoscopy. While in case of an emergency situation, management should take precedence with all standard infection control measures as for COVID-19 suspected cases.

Safety of the health care workers: Before starting a service or test, ensure the availability of staff, space, and PPEs. All efforts should be made to segregate and stagger the services in these areas to avoid overcrowding. Table I summarizes risk of infection with different services/procedures, when these can be resumed, and required level of PPE. Healthcare workers should inform hospital authorities in case they develop ILI symptoms for appropriate action and avoid going to patient care facilities.

\section{Care of Chronic Respiratory Diseases}

Asthma [4]: All patients should continue inhaled steroids as before. A short course of prednisolone may be advised early for asthma exacerbation to prevent hospital visit. Avoid nebulization as far as possible, using metered dose inhaler (MDI) with spacer instead. Treatment for allergic rhinitis may be continued as before.

Tuberculosis (TB) [5,6]: Continue anti-tubercular treatment as before. Modify regimen for multi-drug resistant tuberculosis to all oral drugs to reduce hospital visit. If a child with tuberculosis develops COVID-19, continue TB treatment.

Cystic fibrosis [7,8]: Continue usual care as before. Minimize the use of nebulizer. Recognize and treat $\mathrm{CF}$ exacerbations promptly and aggressively with oral or, if required, parenteral therapy at home. Hospitalize only for severe exacerbations.

Interstitial lung disease (ILD): Continue treatment for follow-up cases of ILD. For new cases, start immunosuppressive therapy only if absolutely necessary.
Repeat blood test and imaging only if it is required to adjust the therapy.

Suppurative lung diseases: Continue usual treatment as before. Treat the exacerbation early and aggressively, preferably at home.

\section{Diagnostic and Therapeutic Procedures}

\section{Diagnostic Procedures}

Pulmonary function tests (PFTs) [9]: PFTs should be performed only in situations where the available information is not adequate to make certain diagnostic or therapeutic decisions and PFT can resolve these. These should be timed with other essential visits of the patient. Use disposables as much as possible and use viral filter for PFTs. Disinfect the equipment as per advice of manufacturer.

Chest imaging: There is a need to optimize imaging procedures after a detailed discussion, and routine imaging should be avoided. Prior discussion with radiologist can help to keep the procedure focused, essential, and finish in minimum possible time. A digital copy of the imaging procedure can decrease the need for visits to collect and show the reports.

Gastric aspirate and induced sputum: Restricting the testing to those with clinical or radiological chest findings will optimize the yield as well as lessen the risk by avoiding unnecessary cases. Where possible, gastric aspirate may be preferred over induced sputum. During induced sputum procedure, premedication with salbutamol may be done with MDI and spacer, and thus restricting nebulization only for hypertonic saline medication.

Flexible bronchoscopy [10,11]: It should be performed only when alternate diagnostic modalities have failed to reach a diagnosis and when bronchoscopy findings will offer an immediate management. There should be minimum staff in bronchoscopy suite and child should be well sedated during procedure to avoid excessive crying or coughing.

Tuberculin skin test (TST) and skin prick test (SPT): TST can be administered by staff taking all precautions (including PPE) and should be combined with essential visits to decrease the visits to the hospital. SPT has limited role in management of asthma and may be deferred till control of pandemic.

High speed video microscopy (HSVM) and electron microscopy (EM): These tests can be considered with flattening of curve as there is no definite treatment for primary ciliary dyskinesia and supportive therapy may be started based on clinical diagnosis. If test is done during 
ongoing pandemic, consider pre-procedure SARS-CoV-2 testing of the patient.

Sweat test and aquagenic wrinkling skin test: These tests should be resumed as delay in diagnosis of cystic fibrosis may increase morbidity. These are not aerosol-generating procedures, though crying of child during procedure may be a potential risk factor for disease transmission.

\section{Therapeutic Procedures}

Inhalation therapy: There must be a balance between risk of transmission of disease and the negative effect of not delivering inhalation therapy. Nebulization should be used only, if there is no alternative or in case of lifethreatening asthma. Use breath actuated nebulizers, mouth piece interface instead of face mask, and filters or one way valves whenever feasible. In pediatric intensive care unit (PICU), use mesh nebulizers (if available) and filters in expiratory limb of ventilator. Wherever possible, use MDI with spacers.

Airway clearance technique (ACT) services: ACT services should be resumed to prevent progression of chronic suppurative lung diseases. Patients already on ACTs can share videos with healthcare providers for assessment and corrective actions [12]. ACTs to newly diagnosed patients should be taught with all general measures with the help of videos of ACTs. At home, ACTs should be performed in separate well-ventilated room and nebulizer and ACT devices should be cleaned/ disinfected thoroughly after each session of ACT. ACTs in acute care setting should be those not requiring disconnection from ventilator and it should be followed by closed suction [13].

\section{Emergency Care}

Some milder emergency visits may be obviated by using tele-consultation. Divide pediatric emergency department (ED) into clinical triage zone (for quick assessment for sickness level, ILI, and SARI), quarantine zone (to keep suspected COVID-19 till report available) [14]. Ensure rapid turn-over of COVID testing to segregate and channelize cases rapidly from ED (positive cases to COVID wards or home isolation for mild cases, and negative ones to wards/PICU).

Use minimal possible flow for high flow nasal canula (HFNC) and preferably use mask over canula. For noninvasive ventilation, use NIV with non-vented masks and use a viral filter in expiratory limb of the circuit. If using a vented mask, may try to apply a 3-ply surgical mask in front of NIV mask. If intubation is required, use rapid sequence technique, keep minimum number of persons, and intubation should be performed using cuffed tube, by the most experienced person. Personnel involved in CPR should be kept to minimum necessary.

In acute severe asthma management, use nebulization minimally, mainly for life threatening acute asthma; medications should preferably be given using MDI and spacers, where possible [15].

\section{CONCLUSIONS}

The ongoing SARS-CoV-2 pandemic is hampering nonCOVID services including pediatric pulmonology. As the pandemic is unlikely to go away in near future, we need

\section{Box I Gaps in Knowledge and Research Needs}

- The literature/evidence is limited regarding viability of SARS-CoV-2 on different objects/surfaces, and risk of transmission of disease by using different type of oxygen providing devices and by various aerosol generating procedures. It is also unclear how much transmission risk exists when infants cry.

- There is a need for a study to assess the risk of transmission among HCWs in different clinical areas like OPD, in-patient, ED, and pediatric intensive care units, different ACTs, during various PFTs in children, during flexible bronchoscopy, and for a particular radiological procedure.

- Long term impact of COVID-19 on lung health in normal children and children with chronic respiratory diseases need to be evaluated.

- Drug interactions of drugs for TB and COVID-19 need to assessed. Effect of COVID in children with TB is not clearly known.

- Remote testing including mobile based 6-minute walk test with oximetry monitoring, one-minute sit to stand test and 40 steps test and assessing physiological parameters and attempting pulmonary rehabilitation using telemedicine may be explored for exercise testing.

- There is lack of study documenting presence or absence of SARS-CoV-2 in sweat in adults or children and risk of transmission by sweat.

- There is lack of studies regarding remote reading of TST. 
to resume non-COVID services. It is a consensus statement to guide health care professionals to restart the pediatric pulmonology services. We recommend general measures to reduce hospital visits, to reduce cross infections etc. There is need to adapt specific measures for various clinical, diagnostic and therapeutic services. Safety of health care workers is of paramount importance. There are still may gaps in knowledge regarding COVID19 and need further research.

Disclaimer: The group recognizes the fact that the understanding of COVID-19 is evolving as it is a new disease and the pandemic is a dynamic process. This consensus statement, therefore, may become outdated, changed or redundant over time as more evidence is generated. Hence, it is recommended to that the guidance are followed in line with the directives and other statutory guidelines adopted by local authorities and the medical societies. It is an executive summary of the position statement. The full position statement is available at $h$ ttp://pedspulmcar.aiims.edu/Login/Login.aspx

Contributors: SKK, KRJ, RL: Conception of idea; KKB, JIB, RRD, ND, JJG, JPG, KMG, SG, KRJ, PK (Pawan K), AK, PK (Prawin K), VK, AP, JLM, VHR: Reviewed the literature, prepared initial draft of manuscript; RL, JLM, VS, SKK, KRJ: Reviewed the manuscript and revised it critically for important intellectual content; KRJ: co-ordination and collation, corresponding author; All authors had critically revised and approved the final version of the manuscript.

Funding: None; Competing interest: None stated.

\section{REFERENCES}

1. World Health Organization. Infection prevention and control during health care when novel coronavirus $(\mathrm{nCoV})$ infection is suspected [Internet]. Available from: https://www.who.int/ publications-detail-redirect/10665331495. Accessed June 6, 2020.

2. World Health Organization. Risk Assessment and Management of Exposure of Health Care Workers in the Context of COVID-19: Interim Guidance [Internet]. Available from: https://www.who.int/publications-detailredirect/risk-assessment-and-management-of-exposure of-health-care-workers-in-the-context-of-covid-19interim-guidance. Accessed June 6, 2020.

3. Telemedicine.pdf [Internet]. Available from: https:// www.mohfw.gov.in/pdf/Telemedicine.pdf. Accessed May 20, 2020.

4. NICE. COVID-19 Rapid Guideline: Severe Asthma. [Internet]. NICE. Available from: https://www.nice.org. uk/guidance/ng166. Accessed June 22, 2020.
5. World Health Organization. Information Note Tuberculosis and COVID-19 COVID-19: Considerations for tuberculosis (TB) care. 2020. Available from: https:// www.who.int/tb/COVID_19considerations_tuberculosis_ services.pdf. Accessed July 20, 2020.

6. Stop TB Partnership. TB and COVID-19 [Internet]. Available from: http://www.stoptb.org/covid19.asp. Accessed June 4, 2020.

7. NICE. COVID-19 Rapid Guideline: Cystic Fibrosis [Internet]. Available from: https://www.nice.org.uk/ guidance/ng170. Accessed June 3, 2020.

8. CF Foundation. COVID-19 community questions and answers [Internet]. Available from: https://www.cff.org/ Life-With-CF/Daily-Life/Germs-and-Staying-Healthy/ CF-and-Coronavirus/COVID-19 -CommunityQuestions-and-Answers/. Accessed June 3, 2020.

9. Recommendation from ERS Group 9.1 (Respiratory function technologists/Scientists) Lung Function Testing During COVID-19 Pandemic and Beyond [Internet]. Available from: https://www.artp.org.uk/write/Media Uploads/Standards/COVID19/ERS_9.1_Statement_on_ lung_function_during_COVID19_Version_1.0.pdf. Accessed June 6, 2020.

10. Pollaers K, Herbert H, Vijayasekaran S. Pediatric microlaryngoscopy and bronchoscopy in the COVID-19 era. JAMA Otolaryngol Neck Surg. 2020;146:608-12.

11. Wahidi MM, Lamb C, Murgu S, Musani A, Shojaee S, Sachdeva A, et al. American Association for Bronchology and Interventional Pulmonology (AABIP) Statement on the Use of Bronchoscopy and Respiratory Specimen Collection in Patients with Suspected or Confirmed COVID-19 Infection. J Bronchol Interv Pulmonol. 2020, March 18. [E-pub Ahead of Print].

12. British Thoracic Society COVID-19: information for the respiratory community. Better lung health for all [Internet]. Available from: https://www.britthoracic.org.uk/about-us/covid-19-information-for-therespiratory-community. Accessed June 5, 2020.

13. Thomas P, Baldwin C, Bissett B, Boden I, Gosselink R, Granger CL, et al. Physiotherapy Management for COVID-19 in the Acute Hospital Setting: Clinical Practice Recommendations. J Physiother. 2020;66:73-82.

14. Yen MY, Lin YE, Lee CH, Ho MS, Huang FY, Chang SC, et al. Taiwan's traffic control bundle and the elimination of nosocomial severe acute respiratory syndrome among healthcare workers. J Hosp Infect. 2011;77:332-7.

15. Levin M, Morais-Almeida M, Ansotegui IJ, Bernstein J, Chang YS, Chikhladze $\mathrm{M}$, et al. Acute asthma management during SARS-CoV2-pandemic 2020. World Allergy Organ J. 2020;100125. 